\title{
OPAQUE SPIRAL DISKS: SOME EMPIRICAL FACTS AND CONSEQUENCES
}

\author{
EDWIN A. VALENTIJN \\ European Southern Observatory, Karl-Schwarzschild-Str. 2 \\ D - 8046 Garching bei München
}

\section{INTRODUCTION}

A recent analysis of the surface brightness profiles of a complete sample of 9381 spiral galaxies extracted from ESO-LV (Lauberts and Valentijn, 1989) showed that many galaxy disks of especially $\mathrm{Sb}-\mathrm{Sc}$ galaxies are opaque (Valentijn, 1990). This paper studied how the observed surface brightness $\mu^{o b s}$ varies as a function of the observed axial ratio $a / b$, by fitting the data of samples of spirals with

$$
\mu^{o b s}=\mu^{f a c e}-2.5 C \log (a / b),
$$

assuming the $a / b$ to give the inclination angle of the disks to the line of sight. A sample of galaxies that were fully transparent at the particular radius used for the test would have $C \simeq 1$, while $C<\sim 0.25$ signifies opaque conditions, the transition value being heavily dependent on the spatial distribution of the absorbing material and the effect of multiple scatterings (Bruzual et al. 1988). The most frequentlyused $C$ values range from 0.5-0.9 (Holmberg 1975, de Vaucouleurs et al. 1976, Sandage and Tammann 1981). However, Valentijn (1990) derived $C$ values well below 0.25 for large samples of spirals throughout the galaxy disks; this result is not significantly affected by selection effects in either magnitude, angular diameter or axial ratio, nor by the presence of bulges (at least for types $\mathrm{Sb}$ and later).

In an interesting study, Disney et al. (1989) discussed the possibility of opaque disks on the basis of the IRAS infrared flux. However, the IRAS detectors were most sensitive to warm $(\sim 60 K)$ dust components, with mostly cirrus-like distributions. Applying their models to the ordinary ESO-LV disks, the detected IRAS fluxes or the upper limits are too low to make them opaque by this component, unless large bolometric corrections from the IRAS-band to the total FIR-band are applied. Such large bolometric corrections actually represent the introduction of a second cooler component, which a priori need not have any direct physical association with the warm component, neither to follow its spatial distribution. In fact, if the cirrus were to follow the spatial distribution of the stellar component, then the Disney et al. models would have great difficulties in explaining the observed exponential visual light profiles in otherwise opaque disks. A recent study of Chini et al. (1986) demonstrated a substantial sub-mm signal in $18 \mathrm{Sb}-\mathrm{Sc}$ 's, with a typical black-body temperature of $16 K$. Adopting a $\lambda^{-2}$ dependency of the grain opacities (not applied by Disneyet al.) an average $L^{\text {cold }} / L^{\text {warm }}$ luminosity ratio of $\sim 1.4$ was obtained, emphasizing the potential importance of the cool component $\left(L^{\text {warm }}\right.$ 
from IRAS data). Lacking detailed knowledge of both the grain opacities at sub$\mathrm{mm}$ wavelengths (e.g. Draine 1989) and the actual temperature distribution of the cold material, it is yet not possible to predict from the observed sub-mm continuum flux the effective total visual light cross-section of the cool component.

Here, I review the results for the Sb's and the Sc's, as obtained from the analysis of the optical ESO-LV data, and discuss the implied constraints for the properties of the absorbing components in spiral disks, which leads to an alternative interpretation of flat rotation curves and a revised extinction model for the Galaxy. I will argue that the presently available data are best understood, when in addition to a cirrus-like dust component that causes extinction (i.e. absorption plus scattering) a second component is causing complete obscuration (occultation). This second component could be identified with compact opaque clouds that have a temperature well below the typical IRAS temperature of $\sim 60 \mathrm{~K}$ and a spatial distribution described by an exponential with a scale length larger than that of the stars.

Some key observations of the analysis of the ESO-LV data are:

-key 1: The central Blue surface brightness $\left(\mu_{0}^{B}\right)$ has a very small dispersion of $0.6^{m}-0.7^{m}$ around the mean value (Freeman, 1970).

-key 2: The mean $\mu_{0}^{B}$ increases from 20.5 for Sa's to 22.3 for the latest spiral sub-types (Sd-I's), which also have on average a lower luminosity, but $\mu_{0}^{B}$ is not correlated with the luminosity when considering a given sub-type.

-key 3: The central $C$ values range from -0.18 to +0.10 .

-key 4: Both the $\mu^{B}$ at the half total light radius $r_{e}$ and a profile fit between $r=5^{\prime \prime}$ and the $\mu^{B}=25$ isophote indicate $C$ values in the range 0.08 to 0.22 .

-key 5: The diameter ratio $D_{26} / D_{e}$ does not depend on $a / b$, which directly implies a $C$ value at the $\mu^{B}=26$ isophote similar to that at $D_{e}$.

-key 6: Key 3, 4 and 5 combine to indicate a modest radial variation of $C$. Typical Sb-c disks have $C=0.0$ at the center and $C \simeq 0.25$ at the outer isophotes.

\section{THE PROPERTIES OF THE ABSORBING BODIES}

\subsection{Single layer models}

I will discuss the observations in terms of single layer models (called slabs in Disney et al. 1989); this particular choice will be justified in Section 2.3. A layer containing uniformly distributed stars with spatial density $\eta_{\star}$ and average luminosity per star $\epsilon_{\star}$, and uniformly distributed obscurers with spatial density $\eta_{d}$, each obscurer having an effective cross-section $\sigma_{d}$, has an intensity

$$
I=\epsilon_{\star} \eta_{\star} \lambda\left(1-e^{-\tau}\right)=\epsilon_{\star} \frac{\eta_{\star}}{\eta_{d} \sigma_{d}}\left(1-e^{-T \eta_{d} \sigma_{d}}\right),
$$

and optical depth :

$$
\tau \equiv \frac{T}{\lambda}=T \eta_{d} \sigma_{d}
$$

with $T$ the metric thickness of the layer and $\lambda$ the mean free path; $\lambda=\left(\eta_{d} \sigma_{d}\right)^{-1}$.

$$
\begin{array}{cc}
\text { For } \tau \gg 1: & I=\epsilon_{\star} \lambda \eta_{\star}=\epsilon_{\star} \frac{\eta_{\star}}{\eta_{d} \sigma_{d}} . \\
\text { For } \tau \ll 1: & I=\epsilon_{\star} T \eta_{\star} .
\end{array}
$$


The relation between optical depth and absorption in magnitudes, $A$, is

$$
A=2.5 \log \left(\frac{\tau}{1-e^{-\tau}}\right)
$$

while the face-on optical depth $\tau_{0}$ can be derived from the observed $C$ values by evaluating numerically:

$$
C \log (a / b)=\log \left(\frac{1-e^{-(a / b) \tau_{0}}}{1-e^{-\tau_{0}}}\right) .
$$

Since $\tau=(a / b) \tau_{0}$, the previous two equations can be applied to transform between $C, A, A_{0}, \tau$ and $\tau_{0}$.

\subsection{The central regions: $\tau \gg 1$}

Low $C$ values obtained for the very central regions should always be assessed most critically, because it is very difficult to quantify the contribution of nuclei or small central spheroidal components, whose effect will be to pull the $C$ values towards lower numbers. However, for a sub-sample of pure exponential disk systems the low central $C$ values were reproduced, and especially for Sb's and Sc's their average $\mu_{0}^{B}$ hardly differed from the total sample. Thus, there is strong evidence that the observed low central $C$ values are associated with the central parts of the disks, indicating opaque conditions. Equation (4) then implies that the central flux is basically determined by the star-to-obscurer density ratio, which leads to

- Concl. 1: the small dispersion of $\mu_{0}$ (key 1) reflects a limited range in the starto-obscurers density ratio in different galaxies, which ratio however decreases for the later types (key 2). This is in opposition to previous suggestions that key 1 results from a constant surface density of stars in transparent systems, which implicitly invokes a constant metric disk thickness, $T$ (van der Kruit, 1987). This latter hypothesis would also require a constant $T$ for disks that vary over a factor of 100 in intrinsic diameter. Detailed modeling indicates that the observed negative $C$ 's can occur when additional extinction by surrouding semi-transparent regions (along the line of sight) acts on the light emitted by fully opaque central regions.

\section{3. $C=0.2$ disks: no $\tau \gg 1$ layers}

The low $C$ values at the half total light radii (key 4) and the evidence for similar $C$ values at the outer isophotes (key 5 ) constrain the spatial distribution of the obscuring component. A disk with $C=0.2$ could originate from one of the following configurations:

i) A single layer of dust and stars as described in Section 2.1 with $\tau_{0} \sim 1.3$,

ii) A single layer of dust and stars with $\tau \gg 1$, in which the effect of multiple scatterings brighten the surface brightness at larger inclinations (Bruzual et al. 1988), iii) A two layer situation in which $80 \%$ of the flux originates from a $\tau \gg 1$ layer and the remaining part from a transparent layer.

However, in case ii), the total dust masses involved would have to be very high (Section 2.3.2), while for both ii) and iii) the $\tau \gg 1$ layer will have great difficulties in producing an exponential light profile (2.3.1). Interestingly enough, after excluding $\tau \gg 1$, a $C$ value around 0.2 implies that the detected light should essentially 
originate from a single layer à la $i$ ), with $<20 \%$ of the detected flux arising from a surrounding transparent layer. This justifies the application of the single layer equations and leads to

- Concl 2: key 6 implies through (7)that $\tau_{0}=T \eta_{d} \sigma_{d}$ only modestly decreases with radius.

\subsubsection{Scale height and scale length of the obscuring component}

If the scale height $t$ of the obscuring component were much smaller than that of the stars, we would observe $C$ values near unity and not even notice that we miss half of the system. The existence of small $t$ layers can not be ruled out, but there is no observational evidence that requires them. Even if they exist, the observed $C$ must be due to another component.

- Concl. 3: The scale height of the obscuring component must be intermediate or equal to that of the stars.

- Concl. 4: For well-mixed stars and obscurers a $C=0.2$ disk then corresponds to $\tau_{0}^{B} \simeq 1.3$ and face-on $A_{0}^{B} \simeq 0.63^{m}$, via (6) (for $C=0.08: A_{0}^{B} \simeq 1.0^{m}$ ). Formally, these values are lower limits, due to the unknown contribution of stars above the obscuring layer and the effect of multiple scatterings off cirrus-like distributed dust. As pointed out above, these contributions must be small.

The combination of exponential light profiles in otherwise $C=0.2$ disks sets more constraints: equation (2) should now be read as a radial description, with the condition of the modest radial variation of $\tau$ in (3). For $\tau \gg 1$, models with $\eta_{\star} / \eta_{d}$ constant with radius will have constant surface brightness profiles. Although some spiral arms of ESO-LV spirals exhibit flat surface brightness profiles, many $C=0.2$ disks are observed to have an exponential light profile. So both for $\tau \gg 1$ models and for the favoured $\tau=1.3$ situation:

- Concl. 5: the ratio of the density of the stars to that of the obscuring bodies (multiplied by their cross-section) should decrease with radius in $C=0.2$ disks with constant or modestly varying $\tau$ in order to explain their exponential light profile. This can be formalised in terms of the scale length of the obscuring component, $\alpha_{d}$, compared to that of the stars, $\alpha_{\star}$, and that of the observed light, $\alpha_{o b s}$. If the stars have $\eta_{\star}(r)=\eta_{\star}(0) \exp \left(-r / \alpha_{\star}\right)$ and the obscurers have $\eta_{d}(r)=\eta_{d}(0) \exp \left(-r / \alpha_{d}\right)$, while the observed light profile is $I(r)=I(0) \exp \left(-r / \alpha_{o b s}\right)$, then substituting this in (2) results in:

$$
\alpha_{o b s}=\frac{\alpha_{\star}}{1-\alpha_{\star} / \alpha_{d}}
$$

assuming the obscurer's cross-sections do not vary with radius.

- Concl. 6: (8) formalises how the observations (i.e. $\alpha_{o b s}>0$ ) require the scale length of the obscuring component to be larger than that of the stars $\left(\alpha_{d} / \alpha_{\star}>1\right)$.

\subsubsection{The nature of the obscuring bodies}

The observed nominal value of $\tau$ at the half total light radius corresponds to the local determination of the product: $\tau=T \eta_{d} \sigma_{d} \simeq 1.3$. This can be used to estimate the total mass of the obscurers if they were composed of cirrus-like dust. When describing the macroscopic properties of cirrus with an exponential radial density 
distribution, and the more uncertain microscopic properties of the grains with mass density $s$ and a ratio $Q$ between the effective cross-section $\sigma_{d}$ and the geometrical cross-section, a rough total mass interior to a galactic radius $r$ due to grains of radius $R$ (in units of $1 \mu \mathrm{m}$ ) can be estimated according to:

$M(<r)=T m_{d} \int_{0}^{r} 2 \pi r \eta_{d}(r) d r=4.2 \cdot 10^{6} e^{1.67 n \alpha_{\star} / \alpha_{d}} \tau_{n e} s_{g / c m^{3}} Q^{-1} R_{1 \mu m} Y_{1 k p c^{2}}\left(M_{\odot}\right)$

with $Y(r)$ in units of $k p c^{2}: \quad Y=\alpha_{d}^{2}-\alpha_{d}\left(r+\alpha_{d}\right) e^{-r / \alpha_{d}}$,

and $\tau_{n e}$ the measured value of $\tau$ at a particular radius $n \times r_{e}$. If the radial density distribution of cirrus follows that of the stars, as the IRAS data suggest (e.g. Wainscoat et al., 1987 in N891, N4565 and N5907, and Bloemen et al. 1990 in the Galaxy), for instance with $\alpha_{\star}=\alpha_{d}=4 k p c$, and using $s=2.5, Q=2$, and the typical post-IRAS grain sizes in the range $R=0.01-0.1 \mu m$ (e.g. Rowan-Robinson 1986, Cox and Mezger 1989) we obtain $M(r<10 \mathrm{kpc})=5.3^{n} \times 0.8-8 \cdot 10^{6} M_{\odot}$ for the mass associated with the small grains in some typical ESO-LV disks. We observed, however (key 5), a modest radial variation of $\tau$ beyond $r_{e}$, for instance out to $r / r_{e}=n=2$. This would then imply dust masses $M(r<10 k p c)=0.2-2 \cdot 10^{8} M_{\odot}$. Noting the well documented star-to-cirrus-dust mass ratio of 1000 to 1 (at least in the solar neigbourhood), we see that the deduced masses are very high and seem acceptable only for ultra-small grains. However, if the ultra-small grains were accompagnied by larger grains or extended to create $\tau \sim 1$ at $n=2.5$ (about the $26^{t h}$ isophote for many ESO-LV disks), the predicted $M(r<10 \mathrm{kpc})$ would exceed plausible values. Evidently, even larger masses will be obtained when the main absorption job is performed by any larger solid bodies.

- Concl. 7: There appear a number of reasons why we should face the possibility that the main absorption is due to another component than grains in a cirrus-like configuration: i) cirrus grains could lead to exceptional dust masses and the typical cirrus temperatures predict IRAS fluxes much higher than is actually observed (see also Introduction), ii) Concl. $5\left(\eta_{\star} / \eta_{d}\right.$ decreasing with radius) or equivalently Concl. $6\left(\alpha_{d}>\alpha_{\star}\right)$ are not consistent with the spatial distribution of cirrus as mapped by IRAS. Although, at larger radii, where the interstellar radiation field will be less intensive, cirrus cooler than that seen by IRAS might produce $\alpha_{d}>\alpha_{\star}$ (solving $i i)$ ), i) still invites to consider alternatives.

Prominent candidates to contribute to the absorption are compact opaque molecular clouds. The fundamental reason why they can be more effective is that the clouds represent large bodies, most likely in a virialized state with virial mass $m_{c l}=3 G^{-1} R v^{2}$, where $R$ is the radius and $v$ the one-dim. velocity dispersion. Solomon et al. (1986) measured $v=(S / 1.01)^{0.5}$, and thus $m_{c l}=700 f S^{2}\left(M_{\odot}\right)$; here $S$ is the metric size dispersion in $p c$ and $f$ a projection factor $(\pi)$. Thus, contrary to individual dust particles, the ratio (cloud-mass/cross-section) is independent of the size, and absorption models involving molecular clouds will be invariant for the chosen cloud dimensions and hence their mass. This geometric difference compared to cirrus dust particles give compact clouds a higher overall absorption efficiency. We can compute the total cloud mass required to give a particular absorption even without knowing the cloud dust content, by using the 
observed size-line-width relation. Issa et al. 1990 pointed out that spatial confusion might seriously affect the size-line-width relation and especially the mass determinations for the largest clouds. However, the size-line-width relation is also observed for the smallest clouds and recently Lee et al. 1990 presented a linear relation between cloud mass estimates from $C O$ data and virial masses.

Assuming the compact clouds obscure the background out to their gravitational radius $R=q S$, with $q=2.5$, Spitzer 1969 , (note, $S$ was defined as size dispersion) the exponential distribution of $\eta_{d}$ and the fixing of the optical depth at $n \times r_{e}$ lead to the following expression for the total obscuring mass in the form of molecular clouds in a disk within radius $r$ :

$$
M(<r)=T m_{c l} \int_{0}^{r} 2 \pi \eta_{d}(r) d r=2.2 \cdot 10^{8} f q_{2.5}^{-2} \tau_{n e} e^{1.67 n \alpha_{\star} / \alpha_{d}} Y_{k p c^{2}}\left(M_{\odot}\right),
$$

with $Y$ in units of $k p c^{2}$ as in (10). This expression for $M(<r)$ can be used to compute the required obscurers mass from the observed photometric parameters $\alpha_{\star}$ and $\tau_{n e}$ for a given $\alpha_{d}$. The main uncertainty in computing $M(<r)$ using (11) relates to deviations from the assumed spherical symmetry of the clouds and corresponds to different values of the constants $f$ and $q$. However, since both Solomon et al. and Lee et al. find the clouds to conform to a virialized state the choosen values of the constants might be quite correct. For $\alpha_{\star}=4 k p c$ and $\alpha_{d}=$ $6-16 k p c$ a $\tau_{e}=1.3$ disk would need to contain $3-4 \cdot 10^{10} M_{\odot}$ of compact clouds within $r=8 k p c$, if they were to do the main absorption job. This is a large mass, but since this is for the situation with $\alpha_{d}>\alpha_{\star}$, this mass will only modestly increase with increasing $n$, in contrast to the cirrus models with $\alpha_{\star}=\alpha_{d}$. Note that a major portion of the total cloud mass corresponds to the systematic difference between cloud masses from $C O$ data and those resulting from the virial theorem, the latter being a factor 3.5 higher.

- Concl. 8: Compact opaque clouds are good candidates to provide a substantial contribution to $\tau \simeq 1.3$ in ordinary $S b$-Sc disks.

\section{FLAT ROTATION CURVES - NO DARK HALOES REQUIRED}

The analysis of the rotation curves in a number of $\mathrm{Sb}-\mathrm{Sc}$ spirals has led many authors to suggest the existence of massive dark haloes (see van Albada and Sancisi 1986 , for a review). The observed radial luminosity profile with its associated $\alpha_{o b s}$, together with an assumed constant $M / L$ is used to set the mass distribution of the disk. In the so-called maximum disk fits the maximum dynamical mass is put in the stellar disk component, while the remaining mass-discrepancy is assigned to a halo. Alternative solutions with a radially increasing $M / L$ in the disks were discarded, lacking any physical or observational justification. The resultant $M / L$ ratio of the disk is only a side product and not essential. It formerly appeared that the maximum disk fits were resistent against any effects due to extinction. Firstly, if there were more extinction $L$ would go up and correspondingly the $M / L$ would go down, changing only the side product of the fits and not the dynamical mass $M$ itself; further, if there were more extinction it was assumed to be more concentrated towards the centre, and this would only increase the mass-discrepancy. However, - Concl. 9: Concl. $6\left(\alpha_{d}>\alpha_{\star}\right)$, or equivalently concl. $5\left(\eta_{\star} / \eta_{d}\right.$ is decreasing with radius) indicate that the fractional contribution of the obscurers to the projected 
mass density increases with radius.

Irrespective of the true nature of the obscuring bodies, (8) clearly indicates that the true scale length of the stellar distribution can be smaller than the observed value, while $\alpha_{d}$ can be larger, and if the obscuring bodies carry mass, then the apparent total $M / L$ ratio in the disks will increase with radius.

Motivated by these results, González-Serrano and Valentijn (1990) fit the rotation curves of two Sc's previously thought to have very massive haloes. These fits were done with one of the standard codes used for maximum disk fits (Begeman 1987), but now dis-abling the halo component and substituting instead a second disk component. With surprisingly few iterations, combinations of $\alpha_{\star}$ and $\alpha_{d}$ were found that reproduced both the observed $\alpha_{o b s}$ through (8) and the rotation curves (Fig 1). The iterations essentially improved the fit to the steep central parts.
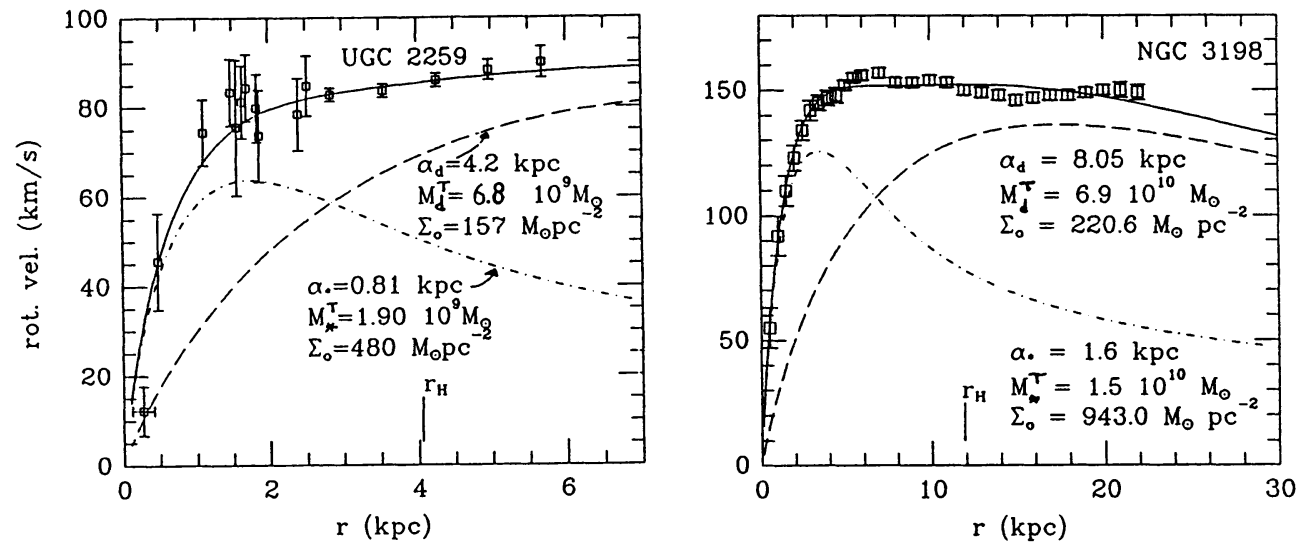

Fig. 1. Rotation curve fits with a two-disk model

The total mass out to the Holmberg radii, involved in the second (obscuring) component exceeds that of the first (stellar) component by a factor of 2-3, quite a large ratio; since here we deal with the most mass-discrepant Sc's with large HI fluxes, these factors do not necessarily represent a standard value.
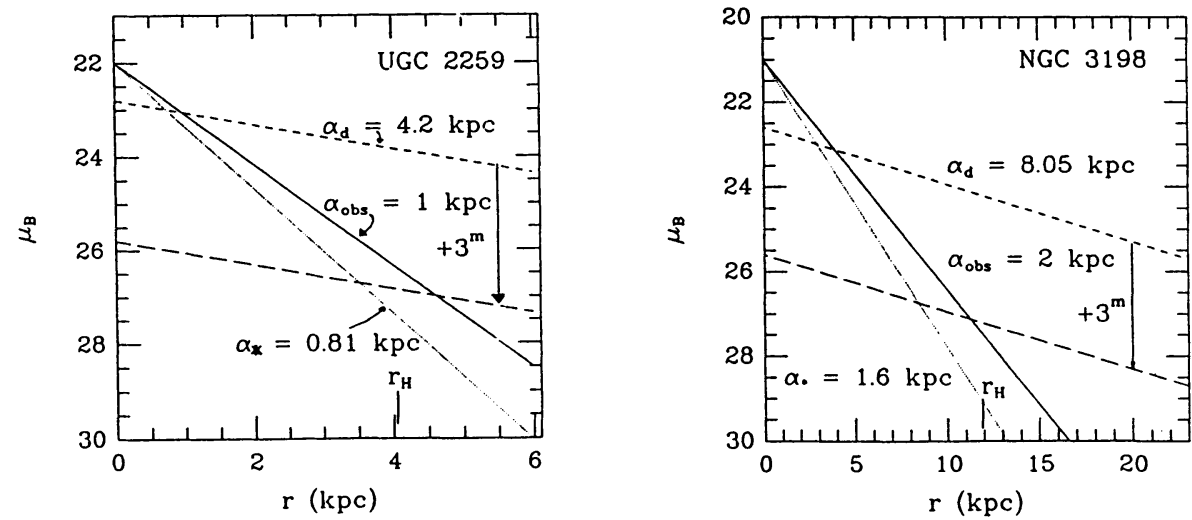

Fig. 2. Hypothetical surface brightness profiles of the two disk components that fitted the rotation curves of UGC 2259 and NGC 3198 
- Concl. 10: A two-disk model with one component representing the visible stellar mass, the second component representing the mass in the obscuring component, can reproduce the observed rotation curves, with the inter-relation between the two components in agreement with the above listed contraints on absorption in $C=0.2$ disks.

Fig. 2 gives an impression of the mass distribution corresponding to the solutions of the fits. This is displayed in units of surface brightness, arbitrarily taking a similar intrinsic $M / L$ for both components. Obviously, the second obscuring component must have a much larger $M / L$ than the stellar component and Fig. 2 demonstrates that, were the second component to contain stars that it self-obscures, an internal extinction of $\geq \sim 3^{m}$ would make it totally negligible in terms of surface brightness; simultaneously, in the case of a $C=0.2$ disk, the effective absorption of the disk due to obscuration can be $\sim 0.63^{m}$.

The solutions for $\alpha_{\star}$ and $\alpha_{d}$ from the rotation curve fits can be substituted into the disk mass equation, (11), to evaluate the mass of the obscuring component, when it consists of compact clouds. Most strikingly, the mass determinations from the dynamical studies (i.e. rotation curve fits) differ less than 10-20\% from the completely independently derived values from photometric studies (i.e. the mass required to make the disks have $\tau_{e}=1.3$ due to compact clouds). Also the central projected mass densities differ by less than 10-20\% between the two methods. Note, that the two components used in the fit represent the prime emission and absorption parts of the single layer model, which should in reality be dynamically coupled and are thus not plagued by the well-known "disk-halo" conspiracy problem.

The strongest point that led to the introduction of dark haloes was the extent of flat rotation curves from HI data, which for UGC 2259 and NGC 3198 reached, respectively, $\sim 1.5$ and $\sim 2$ times the Holmberg radius. Several arguments can be made to explain these observations in terms of the two-disk solutions. First, the extrapolation of the surface brightness profiles in Fig. 2 shows that if these components extended to the HI radius, they would not be detected at optical wavelengths. Second, if the HI flux distribution of these galaxies is described with an exponential, the corresponding scale lengths are very close to that obtained for the obscuring components from the two-disk fits. Thus it appears that the twodisk solutions matches a configuration in which 'dark' matter is spatially associated with the HI gas. Physically, this match corresponds to a limited range of the mass ratio of HI-to-obscuring bodies; if this limited range were universal, it would not be surprising that the "classical" flat rotation curve spirals, which come from $H I$ selected surveys, also contain the most "missing" mass.

\section{THE GALAXY}

\subsection{A special Galaxy, a local hole in the ISM, or a typical $C=0.2$ disk?}

If the Galaxy were like ESO-LV Sbc disks with $\tau \simeq 1.3$, then at the location of the Sun, about two scale lengths from the center, we would expect a total face-on extinction of $A_{B} \sim 0.6^{\mathrm{m}}$. Disney et al. 1989 summarized that a value of $\tau \simeq 1$ (corresponding to $\sim 0.5^{m}$ ) would be allowed by observational data taken at low 
latitudes. The actual extinction to the Poles is subject to debate, with estimates of the total disk extinction ranging from $0.0-0.3^{m}$, which values do not really match the $0.6^{m}$ suggested by ESO-LV data. A local hole in the ISM could be the explanation ( $c . f$. Cox). Alternatively, the Galaxy could be special and contain less obscurers than $C=0.2$ disks. However, all the Galactic extinction studies trace the effect of optically thin cirrus, either by using IRAS data (Boulanger and Pérault 1988) or by measuring the reddening of detected objects. The present work suggests a substantial contribution by occultation due to cool compact opaque clouds, which would be essentially un-noticed by such studies. It seems interesting to explore in more detail whether compact clouds could, at the location of the Sun, result in face-on extinctions similar to those seen in typical ESO-LV Sbc's. Most Galactic extinction studies, either based on reddening (Burstein and Heiles 1978) or on galaxy counts (Shane and Wirtanen 1967), had severe problems matching the Northern and Southern Galactic hemispheres, essentially because of the different origins of the data. The ESO-LV survey has the advantage of covering latitudes between $b=-90^{\circ}$ and $b=+45^{\circ}$ with homogenously acquired data.

\subsection{A new extinction map}

In Fig 3, an extinction map determined from the average reddening of ESO-LV galaxies is reproduced.

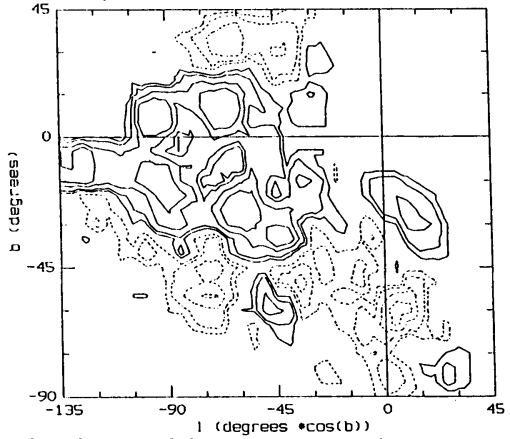

Fig. 3. Reddening map constructed from the central $(B-R)_{10^{\prime \prime}}$ of 11847 galaxies. For each object, the deviation from the mean value of objects with similar morphological type was computed. Then, with a typical $5^{0}$ resolution the average relative reddening was derived. Contour values are $\Delta(B-R)= \pm 0.025,0.05,0.1,0.15$ negative contours are dashed. Note, $A_{B}=2.30 E(B-R)$.

Although the reddening map has a coarse resolution, it is clear that it exhibits much more structure than could be described by a simple cosecant law.

- Key 7: Structures in the reddening map perpendicular to the plane are evident.

- Key 8: The reddening map is asymmetric, with more reddening in the South.

- Concl 11: This demonstrates that the Sun is located slightly above the plane of the main dust layer. This is consistent with a study of the Galactic background light, using Pioneer 10 data, in which Toller (1981) deduces that the Sun is $12.2 \pm 2.1 p c$ above the Galactic plane.

\subsection{Counts of ESO-LV galaxies}

The ESO-LV data base also provides the projected surface density of galaxies.

- Key 9: When extracting sub-samples with selection criteria on either $B, \mu$, type, galaxy density in the environment (clusters, groups, free field), or position on the sky (in/out the supergalactic plane), the surface density in the North $\left(b>0^{0}\right)$ is found to be a factor $\sim 1.6$ higher than in the South $\left(b<0^{0}\right)$. 
This density difference was also found in the Shane-Wirtanen counts, but was not taken seriously because of the differences between the data taken in the North and the South and the potentially dramatic effect of the sky-brightness on the cut-off levels of the counts. ESO-LV avoids these problems; its accurate magnitudes allow the construction of a differential count with a Euclidian normalization (Fig 4).

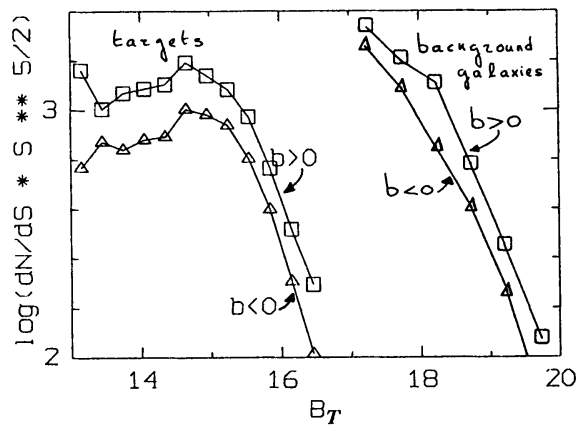

Fig. 4. Differential counts (bin $0.3^{m}$ ) of ESO-LV target and background galaxies normalized with a Euclidian world model. The horizontal parts of the counts of target galaxies indicate that the counts are not hampered by selection effects, which start to operate at about $B=15.5$ for this particular subsample (all types, $\mu_{0}^{B}>21$ ). The surface density difference North - South is evidently a factor 1.6 over the whole horizontal range

Although the North-South density difference is very persistent, no significant galactic latitude dependency was found. Both the galaxy distribution on the sky (e.g. the super-galactic plane) and the reddening map (key 7) show substantial structure perpendicular to the plane, possibly masking any true galactic latitude dependency. The fact is that such a latitude dependency is not observed, and the projected galaxy density has a discrete jump when passing the plane. We are interested in the relative difference in surface density between North and South, and by correlating the relative reddening map of Fig 3 with a map of galaxy counts, we can evaluate how the component that causes the reddening affects the counts. The answer of this exercise is clear:

- Concl 12: The difference in reddening North-South would explain at most a $0.2^{m}$ shift in the un-normalized differential counts, while the observed shift amounts to $\sim 0.8^{m}$ towards fainter magnitudes for the South. This is also illustrated in Fig 5 .

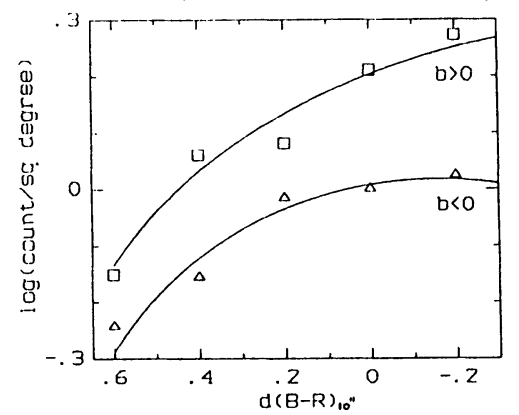

Fig. 5. Galaxy counts versus reddening for North and South. For each field a single count and a single average reddening was computed. The curves show the polynomial regression of the 92 Northern fields and the 313 Southern fields. The points represent the same data binned in $d(B-R)=0.2$. The regressions of the individual curves match the expected effect of extinction via reddening on the counts. However, the offset between the two curves demonstrates that the difference North-South can not be explained with reddening effects (i.e. Concl 12)

- Concl 13: The North-South offset in Fig. 5 also implies that, if this density difference is caused by another obscuring component, then that component should have a very different spatial distribution than the dust that causes the reddening.

Hence, the count difference North-South cannot be caused by reddening due to cirrus-type dust. The possibility remains that it reflects an intrinsic anisotropy. Claims of this nature were presented by Lahav (1987) based on counts of optical galaxies and by Yahil et al.(1986) and Londsdale et al.(1990, preprint) based on 
counts of IRAS galaxies. ESO-LV has a typical depth of $\sim 30 \mathrm{Mpc}$ (with $H_{0}=100$ ) and a local anisotropy on such a scale is momentarily not perceived as unphysical. However, the present notion of the discrete jump when passing through the plane, together with the location of the Sun above the plane and the suggested presence of occulting clouds, should make us most suspicious. In addition, by constructing a similar Euclidian count for a sub-sample of 3000 well-classified background galaxies (total of 60,000), present on the edges of the scans of ESO-LV objects, the NorthSouth difference was extended to $B \sim 19.5^{m}$ (Fig 4 - Jörsäter and Valentijn in preparation), corresponding to a depth of $\sim 200 M p c$. This further points to a Galactic origin for the count difference.

Discarding a high content of sub-mm sized particles that would make the compact clouds opaque at IR wavelength, a discordant note for this interpretation might be the further confirmation of the IRAS galaxies anisotropy at $|b|>50^{\circ}$ (Lonsdale et al.). However, it remains to be proven that this result is not affected by the suggested location of the Sun above the plane (key 8), which if not corrected for in the substraction of the forground emission, with different amplitudes in different directions, might result in an artificial count asymmetry in a similar way as hampered the interpretation of the Shane-Wirtanen counts.

\subsection{A model-The Galaxy as a typical $C=0.2$ spiral disk}

In this section, an extinction plus occultation model for the Galaxy will be explored that obeys the above-labeled conclusions and also reproduces the NorthSouth count difference. If the cirrus-type dust contributes $0.25^{m}$ to a total disk face-on extinction of $0.63^{m}$ (concl. 4), the opaque clouds must contribute the remaining $\sim 0.4^{m}$ or $\tau=0.8$, if they were to resolve the discrepancy. To test this hypothesis, a computer programme was made in which obscuring bodies were randomly distributed in a disk. The apparent face-on $\tau_{0}$ and the fractions $(f(b)$ in percents) of the sky obscured to observers in the disk at $T=0$ were computed, carefully correcting for the effects of overlapping clouds. In Table 1, an abstract is presented of the results of a typical run with $R=5 p c$ spherical clouds, but since the effective obscuration is invariant for cloud dimensions (Section 2.3.2), as long as $\tau$ remains fixed the data in Table 1 could as well represent other or a mix of cloud dimensions. Different diameters only affect the gamble at high galactic latitutes. For $R=12.5 p c$, the model corresponds to a spatial density of $1.6 \cdot 10^{-5} p c^{-3}$, i.e. filling $13 \%$ of the volume with an average cloud separation of $40 p c$. This is a relatively high density, but for instance, correcting the Lee et al. CO survey for distance dependent selection effects, their survey indicates a cloud density $\sim 10^{-6} \mathrm{pc}^{-3}$ above a treshold temperature of $4 K$ for a minimum cloud diameter of $\sim 7 p c$. The authors mention that $68 \%$ of the detected flux is below that treshold temperature and thus the value of $\sim 10^{-6} \mathrm{pc}^{-3}$ is a strong lower limit on the actual cloud density.

Table 1. Model of fractions of the sky $f\left(\right.$ range $\left.b^{0}\right)$ obscured by compact clouds

\begin{tabular}{lcccccccc}
\hline $\mathrm{T}(\mathrm{pc})$ & $\tau_{0}$ & $f(10-20)$ & $f(20-30)$ & $f(30-40)$ & $f(40-50)$ & $f(50-60)$ & $f(60-70)$ & $f(70-90)$ \\
30 & 0.22 & 55 & 23 & 26 & 27 & 0 & 0 & 0 \\
35 & 0.26 & 66 & 28 & 33 & 33 & 0 & 0 & 0 \\
40 & 0.30 & 71 & 31 & 35 & 36 & 14 & 20 & 0 \\
50 & 0.38 & 82 & 41 & 48 & 39 & 28 & 20 & 0 \\
60 & 0.45 & 89 & 49 & 59 & 44 & 31 & 24 & 7 \\
\hline
\end{tabular}


Searching for conditions that produce the observed North-South count difference and adopting a location of the Sun $12.5 p c$ above the plane (concl. 11), the data in Table 1 suggest a solution with a total thickness $T$ of $95 p c$ for the obscuring bodies, corresponding to a $35 p c$ layer to the North and a $60 p c$ layer to the South, with total $\tau=0.71$ and a ratio of obscured sky $f\left(10^{0}-40^{\circ}\right)$ North/South $=1 / 1.54$, (i.e. key 9). The low obscured fractions of the sky at high latitudes (see Table 1), also demonstrate that at these areas the effect of obscuring clouds need not be noticable by the terrestrial observer, but this is subject to strong chance occurences. - Concl 14: A model as tabulated in Table 1 could reproduce the observed NorthSouth count difference, obeying at the same time all the labeled conclusions in this paper. This would make the Galaxy, with respect to the aspects discussed here, a typical $C=0.2 \mathrm{Sbc}$. Applying (11), the total mass of the clouds in this model within the solar circle is $\sim 2 \cdot 10^{10} M_{\odot}$ for $\alpha_{\star}=4 k p c$ and $\alpha_{d}$ in the range $6-20 k p c$; the projected cloud mass density near the Sun is $75 M_{\odot} p c^{-2}$.

ACKNOWLEDGEMENTS. I thank H. van der Laan for his continous encouragement and S. Casertano and M. Rupen for very useful comments on the manuscript.

Albada, van T. S., Sancisi, R. 1986: Phil. Tranc. Roy. Soc., London A320 447-465

Begeman, K. 1987: Ph.D Thesis, Kapteyn Laboratorium, Groningen

Bloemen, J.B.G.M., Deul, E.R., Thaddeus, P. 1990: Astron. Astrophys., 233, 437

Boulanger, F., Pérault, M. 1988: Astrophys. J., 330, 964

Bruzual, G.A., Magris, G.C., Calvet, N. 1988: Astrophys. J., 333, 673-688

Chini, R., Kreysa, E., Krügel, Mezger, P. G. 1986: Astron. Astrophys., 166, L8

Cox, P., Mezger, P.G. 1989: Astronomy and Astrophysics Review, 1, 49

Disney, M., Davies, J., Phillips, S. 1989: Monthly Notices Roy. Astron. Soc., 239, 939-976

Draine, B. T. 1989: The Interstellar Medium in Galaxies, ed. H . A.Thronson and J. M. Shull, Dordrecht, Kluwer

Freeman, K.C. 1970: Astrophys. J., 160, 811-830

González-Serrano, J.I. and Valentijn E.A. 1990: Astron. Astrophys. in press

Holmberg, E. 1975: Stars and Stellar Systems Volume IX, University of Chicago

Issa, M., MacLaren, I., Wolfsdale, A.W. 1990: Astrophys. J., 352,132

Kruit, P.C. van der 1987: Astron. Astrophys., 173, 59-80

Lahav, O. 1987: Monthly Notices Roy. Astron. Soc., 225, 213

Lauberts, A., Valentijn E.A. 1989: The Surface Photometry Catalogue of the ESO-Uppsala Galaxies = ESO-LV, ESO, München

Lee, Y., Snell, R.L., Dickman, R.L. 1990: Astrophys. J., 355, 536

Rowan-Robinson, M. 1986: Monthly Notices Roy. Astron. Soc., 219, 737

Sandage, A., Tammann, G.A. 1981: RSA, Carnegie Institute, Washington

Shane, C.D. and Wirtanen, C.A. 1967: Publ. Lick Obs., Vol. 22, Part I.

Solomon, P.M., Rivolo, A.R., Mooney, T.M., Barret, J.W., Sage, L.J. 1986: in Star Formation in Galaxies Editor C.J. Lonsdale Persson

Spitzer, L. 1969: Astrophys. J., 158, L139

Valentijn, E.A., 1990: Nature, 346, 153

Vaucouleurs, de G., de Vaucouleurs, A., Corwin, H.G. 1976: RC2, Univ. Texas Press

Wainscoat, R.J., de Jong, T., Wesselius, P. R. 1987: Astron. Astrophys., 181, 225

Yahil, A., Walker, D., Rowan-Robinson, M. 1986: Astrophys. J., 301, L1 